\title{
Metodologia de Cuidado Humanitude: Repercussões na atuação dos profissionais em instituição para idosos durante a pandemia da COVID-19
}

\author{
Methodoly of Humanitude Care: Repercussions on the performance of professionals in na \\ institution for the elderly during the COVID-19 \\ Metodología de Humanitude Care: Repercusiones en el desempeño de los profesionales en una \\ institución para personas mayores durante la pandemia COVID-19
}

Kátia Lilian Sedrez Celich

ORCID: https://orcid.org/0000-0002-5166-8444 Universidade Federal da Fronteira Sul, Brasil

E-mail: katia.celich@uffs.edu.br

Rosa Cândida Carvalho Pereira de Melo

ORCID: https://orcid.org/0000-0002-0941-407X

Escola Superior de Enfermagem de Coimbra, Portugal E-mail: rosamelo@esenfc.pt

Mara Ambrosina de Oliveira Vargas

ORCID: https://orcid.org/0000-0003-4721-4260

Universidade Federal de Santa Catarina, Brasil

E-mail: ambrosina.mara@ufsc.br

Liliana Vanessa Lúcio Henriques

ORCID: https://orcid.org/0000-0001-7648-7626 Escola Superior de Enfermagem de Coimbra, Portugal

E-mail: lilianahenriques312@gmail.com

Jeane Barros de Souza

ORCID: https://orcid.org/0000-0002-0512-9765

Universidade Federal da Fronteira Sul, Brasil

E-mail: jeane.souza@uffs.edu.br

Francielly Zilli

ORCID: https://orcid.org/0000-0001-9697-2709

Universidade Federal de Santa Catarina, Brasil

E-mail: franciellyzilli.to@gmail.com

Leoni Terezinha Zenevicz

ORCID: https://orcid.org/0000-0002-0811-6812

Universidade Federal da Fronteira Sul, Brasil

E-mail: leoni.zenevicz@uffs.edu.br

Marceli Cleunice Hanauer

ORCID: https://orcid.org/0000-0002-5798-2709

Universidade Federal da Fronteira Sul, Brasil

E-mail: marceli.hanauer11@uffs.edu.br

Valéria Silvana Faganello Madureira

ORCID: https://orcid.org/0000-0001-7990-3613

Universidade Federal da Fronteira Sul, Brasil

E-mail: valeria.madureira@uffs.edu.br

\begin{abstract}
Resumo
Objetivo: compreender as repercussões do uso da Metodologia do Cuidado Humanitude na vida dos profissionais que atuam em uma estrutura residencial para pessoas idosas de Portugal, durante o enfrentamento da COVID-19. Método: Estudo descritivo, qualitativo, realizado em uma estrutura residencial para pessoas idosas no norte de Portugal. Participaram dessa investigação oito profissionais que apresentavam formação na Metodologia de Cuidado Humanitude e com experiência na assistência ao idoso. A coleta de dados ocorreu de forma online durante os meses de setembro e outubro de 2020, e os resultados foram analisados com base na análise de conteúdo de Bardin. Resultados: Participaram da pesquisa, enfermeiros, assistente social, psicólogo, sociólogo, terapeuta ocupacional e administrador. A problematização dos resultados seguiu duas grandes categorias as quais abordaram as temáticas referentes à consolidação de um ambiente saúdavel no trabalho e a redefinição da gestão do cuidado. Conclusão: Foi possível compreender que a Metodologia de Cuidado Humanitude repercutiu na vida dos profissionais de modo que os mesmos puderam identificar fatores motivacionais, satisfatórios e de valoração do processo de trabalho, assim como, características oriundas tanto dos pilares como dos fundamentos da
\end{abstract}


metodologia, repercutiram de forma positiva na vida dos profissionais diante do enfrentamento das dificuldades experienciadas pela COVID-19.

Palavras-chave: Infecção por coronavírus; Assistência a idosos; Humanização da assistência; Institucionalização; Pessoal de saúde.

\begin{abstract}
Objective: to understand the repercussions of the use of the Methodology of Humane Care in the lives of professionals working in a residential structure for the old people of Portugal, during the confrontation of COVID-19. Method: Descriptive, qualitative study, carried out in a residential structure for the old person in northern Portugal. Eight professionals who were trained in the Methodology of Humane Care and with experience in caring for the elderly participated in this investigation. Data collection occurred online during September and October 2020, and the results were analyzed based on Bardin's content analysis. Results: Study, nurses, social worker, psychologist, sociologist, occupational therapist and administrator. The problematization of the results followed two major categories which addressed the themes related to the consolidation of a healthy environment at work and the redefinition of care management. Conclusion: It was possible to understand that the Methodology of Humanitude Care had repercussions on the lives of professionals so that they could identify motivational, satisfactory and evaluation factors of the work process, as well as orriundas characteristics of both the pillars and the fundamentals of the methodology, reflected positively in the lives of professionals in the face of coping with the difficulties experienced by COVID-19.
\end{abstract}

Keywords: Coronavirus infections; Old age assistance; Humanization of assistance; Institutionalization. Health Personnel.

\begin{abstract}
Resumen
Objetivo: comprender las repercusiones del uso de la Metodología de Atención Humana en la vida de los profesionales que trabajan en una estructura residencial para los ancianos de Portugal, durante el enfrentamiento de COVID-19. Método: Estudio descriptivo y cualitativo, realizado en una estructura residencial para la persona mayor en el norte de Portugal. En esta investigación participaron ocho profesionales formados en metodología de atención humana y con experiencia en el cuidado de personas mayores. La recopilación de datos se realizó en línea durante septiembre y octubre de 2020, y los resultados se analizaron en función del análisis de contenido de Bardin. Resultados: Estudio, enfermeras, trabajador social, psicólogo, sociólogo, terapeuta ocupacional y administrador. La problemática de los resultados siguió a dos grandes categorías que abordaron los temas relacionados con la consolidación de un entorno saludable en el trabajo y la redefinición de la gestión de la atención. Conclusión: Era posible entender que la Metodología de Atención a la Humanidad tenía repercusiones en la vida de los profesionales para que pudieran identificar factores motivacionales, satisfactorios y de evaluación del proceso de trabajo, así como características orriundas tanto de los pilares como de los fundamentos de la metodología, reflejados positivamente en la vida de los profesionales ante las dificultades experimentadas por COVID-19.
\end{abstract}

Palabras clave: Infecciones por coronavírus; Asistencia a los ancianos; Humanización de la atención; Institucionalización; Personal de Salud.

\title{
1. Introdução
}

Em 2019, uma doença surgiu na cidade de Wuhan, na China. Foi descoberto um novo vírus da família coronavírus, denominado Severe Acute Respiratory Syndrome (SARS-CoV-2), que causa a doença Coronavirus Disease 2019 (COVID-19), com alto poder de transmissibilidade, determinando um quadro clínico de infecções assintomáticas, à quadros respiratórios graves (Faro et al, 2020). Devido ao elevado grau de morbidade e mortalidade, chamou a atenção das autoridades sanitárias do mundo. Assim, a Organização Mundial da Saúde (OMS) declarou situação de pandemia e determinou medidas preventivas em todos os continentes (Organização Mundial da Saúde (WHO, 2020).

Não diferente dos demais países europeus, Portugal obedeceu de forma criteriosa as orientações da OMS, cumprindo o distanciamento social, principalmente dos grupos de risco, além das medidas protetivas e uso dos equipamentos de proteção individual (EPI) (Xiang et al, 2020; Ferguson et al, 2020). Logo, diante da conjuntura pandêmica, a necessidade de distanciamento social repercutiu em impactos significativos, principalmente para os idosos por integrarem o grupo de risco. A situação agrava-se no caso de idosos que estão em Estruturas Residenciais para Pessoas Idosas (ERPI), pois representam o principal fator de risco para morbimortalidade da infecção pelo SARS-CoV-2 (Moraes et al, 2020). 
Ainda, o impacto nos profissionais de saúde que atuam na assistência ao público idoso, também, é relevante. Neste caso, estes profissionais, além de expostos aos riscos biológicos, pelas situações cotidianas vivenciadas durante a pandemia, constituem potencial grupo de risco para evoluírem com efeitos adversos na saúde mental, como ansiedade, medo, depressão, insônia e angustia (Kang et al, 2020; Lai et al, 2020). No estudo de Zhang et al, (2020) foi evidenciado que os profissionais de saúde, durante a pandemia da COVID-19, manifestaram sofrimento psíquico, estresse e pressão psicológica pela possibilidade de infectar-se e espalhar o vírus para os idosos sobre seus cuidados e a família, despontando a necessidade de reorganizar a vida profissional, juntamente com a privada e social.

Nesse contexto, interessa entender se a Metodologia de Cuidado Humanitude (MCH) repercute na atuação profissional. Esta metodologia em desenvolvimento desde 1970 por Gineste e Marescotti, inclui três pilares relacionais (o olhar, a palavra e o toque) e um pilar identitário (a verticalidade). Operacionaliza-se por meio de uma sequência estruturada de procedimentos cuidativos humanitude ( $\mathrm{SEPCH})$, que se sistematiza em cinco etapas: 1) Pré-preliminares: consiste no anúncio da presença dos cuidadores, abrindo os canais relacionais, com vistas a evitar abordagem surpresa, com respeito a privacidade e autonomia da pessoa cuidada; 2) Preliminares: possibilita o estabelecimento da relação através do olhar, da palavra e do toque, procurando obter o consentimento relacional por parte da pessoa; 3) Rebouclage sensorial: é a prestação dos cuidados, permitindo manter uma coerente ambiência emocional positiva entre o profissional de saúde e a pessoa cuidada através da utilização dos pilares relacionais; 4) Consolidação emocional: é uma fase em que se promove uma impressão positiva na memória emocional da pessoa cuidada, facilitando a aceitação dos próximos cuidados; 5) Reencontro: é o momento da finalização da relação e da despedida, em que se estabelece o compromisso para um novo encontro e futuros cuidados, evitando a sensação de abandono e de desprezo (Figueiredo, Melo, \& Ribeiro, 2018; Henriques et al., 2019).

A MCH é uma estratégia que realiza o cuidado ao ser humano, acolhendo-o na sua humanidade em qualquer contexto, a qual previne a recusa do cuidado e agitação, uma vez que a pessoa se sente respeitada na sua autonomia e dignidade. Ao ser assertiva, consequentemente também cuida do profissional de saúde (Henrique et al, 2020). Estudo identificou que, a implementação da $\mathrm{MCH}$ ocasiona mudanças na cultura organizacional, pois a partir dela, é possível atribuir intencionalidade à relação de cuidado prestado, tirando o foco central da tarefa realizada, fator este que influenciou na redução dos níveis de burnout investigado entre os profissionais (Pinto, 2019).

Diante do exposto, nossas reflexões são confrontadas pelo contexto emergente da pandemia COVID-19, direcionando os questionamentos para a seguinte problemática: quais as repercussões do uso da $\mathrm{MCH}$ na atuação dos profissionais de saúde, durante o enfrentamento da COVID-19?

Destarte, o estudo objetivou compreender as repercussões do uso da Metodologia do Cuidado Humanitude na vida dos profissionais que atuam em uma estrutura residencial para pessoas idosas de Portugal, durante o enfrentamento da COVID-19.

\section{Metodologia}

Trata-se de um estudo descritivo, com abordagem qualitativa (Yin, 2016), realizado em uma Estrutura Residencial para Pessoa Idosas (ERPI) do Norte de Portugal, onde a MCH já está implantada e em fase de certificação. Nesta instituição residiam 28 idosos, sendo que outros 30 idosos frequentavam o local apenas durante o dia.

A equipe atuante na instituição é constituída por 20 profissionais de diversas áreas. Neste estudo participaram 8 profissionais, selecionados a partir dos seguintes critérios de inclusão: terem formação em MCH e experiência na assistência a idosos na ERPI durante a pandemia da COVID-19. Como critérios de exclusão elencou-se: profissionais que estavam em férias ou em licença durante o período da coleta dos dados. Dos 20 profissionais da instituição, oito atenderam aos critérios de inclusão, sendo que todos foram convidados e aceitaram participar do estudo, não havendo recusas. 
A coleta dos dados ocorreu por meio de entrevistas online, usando a plataforma Zoom, durante os meses de setembro e outubro de 2020. O roteiro da entrevista foi composto de dados sócio demográficos e por uma questão norteadora: “Quais são as repercussões da utilização da $\mathrm{MCH}$ na sua vida como profissional ao cuidar de idosos residentes na ERPI, durante a pandemia da COVID-19?"

Foi adotada como técnica de análise e tratamento dos dados, a categorização proposta por Bardin (2016), a qual é uma modalidade de análise de conteúdo que operacionalmente é constituída por três etapas: pré-análise, análise, tratamento dos resultados e interpretação.

Por tratar-se de uma pesquisa científica envolvendo seres humanos asseguraram-se os princípios éticos e legais postulados na Resolução do Conselho Nacional de Saúde de n 466/12 (Brasil, 2012), sendo aprovada em 18 de julho de 2020 , pelo Comitê de Ética em Pesquisa de uma Universidade Federal do sul do Brasil, sob o parecer CAAE: 33882620.5.0000.5564. Para a realização das entrevistas foi assinada a declaração de consentimento informado, sendo garantido o caráter voluntário da participação e assegurada inteira liberdade de participar ou não da pesquisa. O Termo de Consentimento Livre Eesclarecido foi enviado por email para cada profissional que aceitou participar, após a assinatura e o reenvio a autora principal, as entrevista foram agendadas. Foi também dada a oportunidade de terem acesso à transcrição do verbantim obtido das entrevistas e ao estudo final. Como garantia da confidencialidade e anonimato, foi utilizada para identificar os participantes da pesquisa à letra "E”, que significa Entrevistado, seguido de um número arábico (E1, E2, E3, E4, E5, E6, E7, E8) na ordem cronológica em que as entrevistas aconteceram.

\section{Resultados e Discussão}

Participaram do estudo oito profissionais, sendo seis do sexo feminino e dois do sexo masculino. As idades variaram entre 30 a 50 anos, sendo a maior frequência de 40 anos. Todos os participantes tinham como habilitação curso superior nas seguintes áreas: três enfermeiros, um assistente social, um psicólogo, um sociólogo, um terapeuta ocupacional e um administrador. Em relação ao tempo de atuação na ERPI, dois profissionais tinham seis anos e dois vinte e três anos, sendo que a maior concentração aconteceu entre oito a doze anos.

Da análise de conteúdo das entrevistas emergiram duas categorias 1) Consolidação de um ambiente saudável no trabalho; 2) Redefinição da gestão do cuidado. Em cada categoria foram identificadas duas subcategorias, conforme a Tabela 1.

Tabela 1: Categorias, subcategorias e unidades de registo emergentes das repercussões da MCH na vida de profissionais da saúde na assistência aos idosos.

\begin{tabular}{llc}
\hline \multicolumn{1}{c}{ Categorias } & \multicolumn{1}{c}{ Subcategorias } & Unidades de registo \\
\hline $\begin{array}{l}\text { Consolidação de um ambiente saudável } \\
\text { no trabalho }\end{array}$ & $\begin{array}{l}\text { Motivação, satisfação e valorização com } \\
\text { o trabalho }\end{array}$ & 5 \\
& Redução do estresse profissional & 2 \\
Redefinição da Gestão do Cuidado & Papel dos gestores no cuidado & 3 \\
& Papel dos familiares no cuidado & 2 \\
\hline
\end{tabular}

Fonte: Autores.

\section{Consolidação de um ambiente saudável no trabalho}

Os profissionais que atuaram na ERPI durante a pandemia da COVID-19 mantiveram o foco no cuidado em humanitude, permanecendo fiéis a esse aprendizado. Diante dos desafios vivenciados com a pandemia, os participantes do estudo continuaram a desenvolver de maneira compromissada a assistência aos idosos, sendo que o uso da MCH repercutiu de maneira positiva na atuação profissional, consolidando um ambiente saudável de trabalho o qual pode ser identificado e aqui 
apresentado como duas subcategorias, a da Motivação, satisfação e valorização com o trabalho e Redução do estresse profissional.

\section{Motivação, satisfação e valorização com o trabalho}

A motivação dos profissionais no trabalho não está associada somente à vontade de se manter naquele emprego. Percebe-se que é o sentimento que move as ações na ERPI, influenciando diretamente no comportamento do indivíduo no trabalho, que se relaciona com comprometimento, como evidenciado nos depoimentos a seguir:

"Sei que o nosso coração está no sentido certo, e os nossos óculos não mudam, a forma de olhar, de cuidar, não muda. É isto que me move vir trabalhar e vencer as barreiras diante da pandemia” (E4).

"No dia a dia algumas ações conjuntas e organizadas nos ajudaram a continuar, a levantar de manhãa, $e$ vir $e$ encarar mais um dia e, a nos manter ao lado deles como estávamos antes. Mesmo com as alterações causadas pela pandemia, continuamos a estar próximos, porque eles estão aqui conosco, assim venho encorajada para enfrentar outro dia de trabalho" (E5).

É notório que os alicerces descritos na $\mathrm{MCH}$ são a base das relações. A referida metodologia permite a operacionalização da relação por meio de uma Sequência Estruturada de Procedimentos Cuidativos Humanitude (SEPCH), tendo como objetivo criar um relacionamento positivo, com vistas a alcançar uma sensação de bem-estar para o ser cuidado e também para o cuidador (Henriques et al, 2019). Os profissionais reforçaram que as ações conjuntas e estruturadas os encoraja a retornar no próximo dia ao trabalho, mesmo diante dos mais variados desafios impostos pela pandemia.

A organização do serviço com base na $\mathrm{MCH}$ favorece a preservação da motivação, sentimento este confrontado devido a gravidade da pandemia COVID-19. Estudo aponta que profissionais da saúde são considerados o grupo de maior vulnerabilidade, logo, é compreensivo que sentimentos de apreensão e medo possam ser relatados, reforçando assim a necessidade de um investimento em fatores gerenciais os quais possam potencializar o bem-estar destes profissionais (Oliveira et al, 2020).

O fato de eles terem conhecimento da metodologia de trabalho Humanitude e das boas práticas em que ela se apoia, motiva a sua práxis cotidiana, como descrito a seguir:

"Hoje a comunicação é mais difícil porque temos a máscara que é uma barreira. Tivemos que reinventar o sorriso e começamos a sorrir mais com o olhar. Agora o abraço tem que ser só o nosso, não temos mais a família aqui [...] viver cada momento com os idosos é uma alegria, dá significado a minha vida, o abraço, a palavra doce tem maior significado agora” (E8).

A equipe ao se apropriar dos pilares relacionais (o olhar, a palavra e o toque), que são fundamentos da $\mathrm{MCH}$, compreende que ao alicerçar o cuidado nesses preceitos, sua vida e a assistência em saúde adquire maior significado, promovendo alegria em seu existir. A humanização dos cuidados, por meio da utilização de técnicas relacionais gradativas referentes ao olhar, palavra e toque, evitam abordagens surpresas, o que favorece a assistência, a interação entre cuidador e ser cuidado, promovendo satisfação com o trabalho desempenhado e ampliação da qualidade de vida dos profissionais (Dantas et al, 2017). 
A MCH utiliza técnicas relacionais, caracterizadas pela suavidade, como o toque com ternura, e particularidades muito sutis, em que gestos técnicos e com vinculação são indissociáveis, o que possibilita cuidar e preservar a dignidade da pessoa cuidada (Melo et al, 2017).

Ainda, é interessante observar que a abordagem humana faz sentido a existência dos cuidadores e ela proporciona valorização no trabalho:

"Eu tenho uma abordagem mais humana, eu acho que as pessoas dão maior valor a competência humana, assim temos maior valorização do nosso trabalho, o que me deixa muito grato e com desejo de fazer ainda melhor, ver que as pessoas gostam do meu trabalho" (E1).

"Para mim quando dizem "obrigado", é muito gratificante. Fico muito feliz, sou plenamente feliz com este trabalho" (E7).

A $\mathrm{MCH}$ é uma ferramenta de trabalho que apresenta uma reconhecida riqueza de valores, princípios, normas e pilares que otimiza e qualifica os serviços prestados, consequentemente aumenta a satisfação e motivação dos profissionais envolvidos na relação do cuidar (Costa, Galvão, \& Baptista, 2014). Segundo estudos desenvolvidos por Salyers et al, (2017) os níveis de satisfação no trabalho têm declinado com o passar das décadas, em especial, os que dizem respeito à satisfação no ambiente de trabalho de cuidado a idosos institucionalizados. Ressalta-se que existe uma associação entre satisfação no trabalho e qualidade de vida dos profissionais, sendo indissociável esta conotação (Lima, Gomes, \& Barbosa, 2020).

Os resultados deste estudo demostraram que a satisfação dos profissionais que atuam na ERPI está relacionada a múltiplos fatores, os quais em sua quase totalidade estão interligados a aspectos subjetivos aprendidos na $\mathrm{MCH}$, os quais se materializam nas práticas do trabalho, promovendo motivação para continuar fortalecidos, mesmo diante dos desafios no enfrentamento da COVID-19. O trabalhador se vê na obra que executa, envolvendo-se e dando significado a sua existência por meio das relações desenvolvidas (Lima, Gomes, \& Barbosa, 2020).

Estudo em uma instituição de longa permanência brasileira evidenciou que a falta de qualificação dos profissionais os leva, muitas vezes, a práticas equivocadas em função de estereótipos associados ao envelhecimento, gerando desmotivação e insatisfação com o trabalho, o que compromete a valorização dos profissionais (Roquete, Batista, \& Arantes, 2017).

Neste sentido, fica evidente que balizar o cuidado em uma metodologia que apresenta uma sequência estruturada de procedimentos cuidativos humanitude facilita o desenvolvimento do trabalho. A implementação da MCH para a qualidade da assistência à saúde em uma unidade de cuidados continuados promove a dignidade do ser cuidado, aceitação da assistência, melhora da satisfação profissional e redução da taxa de absenteísmo (Henriques et al, 2019).

A utilização da MCH repercute na vida dos profissionais de forma positiva, expressada a partir da manifestação da satisfação que esses profissionais encontram ao desempenhar seus trabalhos, justamente pela relação que estabelecem com os pacientes. É sabido que o envolvimento com o trabalho, o reconhecimento do sentido das ações realizadas e a consciência de estar fazendo a diferença na vida dos pacientes são fatores geradores de bem-estar, de sentimentos positivos e saudáveis. Já o contrário, a falta de relação afetiva e o não reconhecimento da importâcia do trabalho prestado resultam no desinteresse das práticas laborais (Silva et al, 2020).

Diante das adversidades enfrentadas durante a pandemia da COVID-19, poder contar com um ambiente de trabalho que proporcione relações saudáveis é fundamental. A inexistência de um ambiente de trabalho adequado pode resultar em estresse laboral, comprometer o bem estar físico e emocional, resultando em esgotamento e consequentemente comprometer as práticas de cuidado prestadas (Schultz, et al, 2020). Foi possível identificar a presença de relações saudáveis nas falas onde os 
profissionais não relacionam suas práticas com tarefas a serem cumpridas, e sim com uma abordagem humanizada - onde são capazes de reconhecer o seus valores profissionais - ou seja, como apontado por Tavares, Melo, \& Henriques (2020), a preocupação é em estabelecer uma relação positiva com a pessoa cuidada.

\title{
Redução do estresse profissional
}

O trabalho desempenha papel fundamental nas condições de vida dos indivíduos, é por meio dele que as pessoas se relacionam, oportunizando o convívio social, com desenvolvimento da sua cidadania. No entanto, o ambiente ocupacional pode gerar interferência nas condições de saúde dos trabalhadores, causando tristeza e sofrimento:

\begin{abstract}
"As vezes sentimos como uma bola de neve de negativismo, viemos com nosso plano de cuidados, com a nossa melhor intenção, mas a pessoa não aceita o cuidado, pode até se tornar agressiva conosco e levamos esta carga emocional para o próximo cliente e as vezes até para casa. Ao sair do trabalho nosso coração vai cheio de tristeza! Muitas vezes estamos no limite, há um sentimento de insatisfação, mas ao conversar com um colega que está conosco, logo este sentimento se vai" (E1).
\end{abstract}

"Temos momentos de desânimo, de medo, momentos menos bons, mas conseguimos superar, porque encontramos na humanitude aquilo que ela nos ensinou, um cuidado de modo diferente, mais humano que valoriza a relação com o outro" (E3).

Os depoimentos revelam sentimentos de tristeza, frustração, insatisfação, abalo e desânimo, emoções que podem ter vindo à tona em função do contexto experimentado devido a pandemia da Covid-19 e todo o cenário que ela envolve. Estudo demonstra que as situações vivenciadas pelos profissionais de saúde no enfrentamento a pandemia revelaram a realidade de sofrimento emocional a que estes trabalhadores estão sujeitos, pois muitos tendem a relatar sentimento de culpa, raiva, frustração e tristeza (Duarte, Silva, \& Bagatini, 2021).

No entanto, as falas indicam que o fato de conhecer e utilizar a MCH possibilita a superação dos momentos difíceis. A MCH tem contribuído para que os profissionais de saúde tenham maior conscientização das potencialidades da pessoa cuidada e a compreensão de seus comportamentos, promovendo melhor gestão das emoções, maior intencionalidade na relação e responsabilidade no cuidar, com o despertar do sentimento de dever cumprido e foco no trabalho em equipe (Melo et al, 2019). Tais aspectos favorecem o cotidiano de cuidado e a manutenção de relações harmônicas, com valorização do trabalho em equipe, o que dignifica a pessoa cuidada e os colegas de trabalho.

Ainda, o discurso demonstra que viver em um ambiente agradável e bonito proporciona felicidade e reduz o estresse dos profissionais.

"Tenho que ressaltar que temos aqui um bom espaço físico, uma boa casa, em que as pessoas tem diferentes salas para estar, para fazer suas atividades, temos a horta, o jardim, os carros para os levar a passear, sinto que, todo o espaço que temos tem contribuído para nos sentirmos melhores e mais felizes" (E8).

Este discurso enfatiza a importância de se preservar um príncipio da $\mathrm{MCH}$ relacionado com a transformação das intituições em "lugar de vida e lugar de vontades" (Gineste \& Pelissier, 2008), ou seja deve-se ter em conta a docilidade ambiental, para que os profissionais e as pessoa cuidadas sintam bem-estar, reduzindo os fatores desencadeadores do estress, na medida em que o ambiene atua como auxilio protesico e terapeutico (Cassarino \& Setti 2015). 


\section{Redefinição da Gestão do Cuidado}

A MCH foi implantada em vários países do mundo e está em fase de certificação em Portugal (Figueiredo, Melo \& Ribeiro, 2018). Ela confere segurança aos profissionais, pois sabem o que estão desenvolvendo e como devem realizar a assistência (Henriques, et. al., 2019). No entanto, para seguir uma metodologia orientadora do cuidado é essencial que os gestores das instituições reconheçam nela o seu valor atribuído. Ainda, é primordial que os familiares conheçam e sintam-se seguros quanto as técnicas utilizadas ao deixarem seus entes queridos para serem cuidados em instituições onde a metodologia existe. Dessa forma, para que ocorra uma repercussão positiva da $\mathrm{MCH}$ na vida dos profissionais, foi necessário o envolvimento dos gestores e dos familiares.

A partir deste entendimento, duas subcategorias contemplam essa discussão, a do Papel dos gestores do cuidado e a do Papel dos familiares.

\section{Papel dos gestores no cuidado}

A gestão do cuidado em saúde deve envolver múltiplas dimensões, ajustando-se a cada fase da vida, na direção de buscar o bem-estar, segurança, autonomia e conferir qualidade aos serviços prestados por meio de investigação na formação profissional:

"Explicar para os gestores que é preciso investir no potencial humano, em formação e nas competências profissionais, isto dá qualidade e segurança ao trabalho” (E1).

“As equipes tem permanentemente uma orientação para desenvolver suas atividades e um feedback do seu desempenho e de sua atuação. Somos orientadas para que nunca nos esquecermos daquilo que verdadeiramente importa no cuidado aos idosos que aqui estão, cuidar em humanitude, mantê-los seguros” (E3).

"Todo o suporte na organização do ponto de vista da comunicação, da gestão e da segurança no trabalho são componentes de qualidade no cuidado, não somente os processos, mas também a operacionalização dos processos, como a criação do registro de documentos online em tempo real geram segurança no atendimento” (E4).

"Há um investimento em capacitação, em formação profissional, o componente formativo dá a possibilidade de ser trazido o conhecimento para que as decisões a nível de gestão dos cuidados, gestão dos recursos humanos, gestão de todas as outras áreas para ter uma lógica que funcione melhor” (E2.)

“A partir do momento em que optamos por esta metodologia, ela está em todas as fazes da organização, e isto nos tem ajudado a traçar o nosso caminho, e nos tem mostrado que de fato é este o caminho" (E3).

A MCH é uma ferramenta de gestão que propicia os profissionais de saúde dar intencionalidade à atenção integral dos idosos, nomeadamente em situação de pandemia. Vale salientar que o processo de implementação desta metodologia de cuidado segue quatro etapas: 1) Sensibilização: promove a conscientização dos profissionais sobre a sua prática. Esta formação é teórico-prática, envolvendo os líderes formais e informais; 2) Disseminação: formação na ação, em contexto real de cuidados, envolvendo a direção técnica e os cuidadores; 3) Consolidação: consolida as boas práticas, sendo uma formação dirigida à equipe de gestão estratégica, responsável pela monitorização/avaliação do desenvolvimento do projeto; 4) 
Certificação: valida as boas práticas através de auditorias externas, tendo por base um referencial de cuidado humanitude (Henriques et. al., 2019).

A necessidade de conhecimento, capacitação e formação profissional são fundamentais nesses momentos de crise, e apontadas pelos participantes como fatores importantes na qualidade dos cuidados prestados, proporcionando direcionamento e orientação para a condução da prática. Poder ter como base o envolvimento da gestão e etapas que direcionam a prática de cuidado proporciona segurança para os profissionais conduzirem suas ações. Essas reflexões vão ao encontro de Machado, Pereira, Neto, \& Wermelinger (2020), quando apontam que o conhecimento especializado define condutas técnicas e permite o exercício profissional de forma orientada. As autoras ainda apontam que, durante períodos de pandemia, a organização do serviço com base em orientações científicas é de grande relevância. É apontado que, durante o período de pandemia COVID19, a articulação entre decisões governamentais com a gestão local é fundamental para alcançar respostas adequadas de cuidado (Ventura-Silva et al, 2020).

É imprecsindível que a condução das ações de gestão aliadas ao conhecimento científico possam ser conduzidas também por valores humanizados, ou seja, por uma tecnologia humanizada (Oliveira, 2020). O cuidado humanizado é caracterizado pela interação entre o binômio idoso-profissional (Damaceno, Chirelli, \& Lazarini, 2019). Ao utilizar a MCH verificou uma mudança na cultura organizacional, mais focada na pessoa e na humanização da assistência (Melo et al., 2019).

\section{Papel da família no cuidado}

Na gestão do cuidado, a família há que ser visualizada no contexto multidimensional, valorizando a singularidade das relações interpessoais familiares, dando sentido às vivências e experiências. As relações estabelecidas com os familiares antes da pandemia foram fundamentais para a continuidade do serviço prestado durante o período de distanciamento físico. A forma como a relação com os familiares é constituída, repercute diretamente na atuação profissional, e características da MCH são fortemente identificadas em suas falas:

"Tem sido muito complicado porque somos uma casa aberta, nós recebemos as famílias e as visitas diariamente, em diferentes momentos do dia, não temos limites de horários e nem de visitas, agora tudo está diferente, sentimos a falta de todos eles" (E6).

"As famílias sabem qual é a nossa filosofia e metodologia de trabalho, as vezes algumas famílias percebem melhor do que outras, mas as famílias sabem que é uma coisa importante e que nós temos uma forma de cuidar diferente [...] neste momento da pandemia foi determinante, porque nós sentimos que as famílias continuaram a confiar nos nossos cuidados, apesar de não conseguirem entrar no lar, sentimos um grande apoio e uma grande confiança dos familiares [...] uma preocupação genuína das famílias conosco, com a casa que somos, com o lar que somos” (E3).

Diante do grande risco de contaminação da COVID-19, medidas de prevenção são estratégias necessárias principalmente em residenciais para idosos. Além dos cuidados gerais que contemplam a higiene das mãos, orientações de etiqueta para tossir e evitar tocar o rosto, uma das medidas específicas e fundamentais é a restrição de visitantes, incluindo os familiares (Sunde, \& Sunde, 2020).

Pelo fato da MCH prever acesso aberto para os familiares realizarem visitas, podemos perceber que, ao mesmo tempo em que os profissionais sentem que a pandemia exigiu um distanciamento das visitas presenciais e isso influenciou na rotina de cuidado prestado, os mesmos apontam tranquilidade por saber que conseguiram construir uma relação de confiança com os familiares, e assim conseguem desempenhar a prática profissional de forma tranquila e amparada. 


\section{Conclusão}

O estudo revelou que a Metodologia de Cuidado Humanitude teve repercussões na vida dos profissionais permitindo a consolidação de um ambiente saudável no trabalho, por meio da motivação, satisfação e valorização com o trabalho e redução do estresse profissional, assim como permitiu a redefinição da gestão do cuidado tanto do papel dos gestores do cuidado como do papel dos familiares.

Foram identificados fatores motivacionais, satisfatórios e de valoração do processo de trabalho, assim como, características oriundas tanto dos pilares como dos fundamentos da metodologia de cuidado humanitude que se repercutiram de forma positiva na gestão do cuidado e na vida dos profissionais diante do enfrentamento das dificuldades experienciadas pela COVID-19.

A metodologia evidenciou experiências de cuidado promotoras de bem-estar e satisfação no trabalho dos profissionais de saúde, mitigando os efeitos nefastos da pandemia, nomeadamente a redução do estresse profissional. Neste sentido, torna-se premente a realização de mais estudos que validem, junto aos pacientes e profissioais, os resultados da aplicação da metodologia no contexto de pandemia. Por outro lado, será importante avaliar o impacto da entrevista por videoconferência, necessária na conjuntura de COVID-19, que pode ter limitado o alcance dos objetivos do estudo.

Apesar do interesse de pesquisadores brasileiros pela metodologia, o estudo foi realizado em Portugal, dado ser um dos países onde se tem realizado mais estudos nesta área, sendo que iniciativas de aplicação da MCH no Brasil não são conhecidas. Alude-se à possibilidade da criação de redes colaborativas de pesquisa para a realização de estudos multicêntricos envolvendo outros países, para qualificação e aprimoramento de ferramentas cuidativas que operacionalisem e sistematizem a humanização da assistência com repercussões para os profisionais de saúde e para as pessoas cuidadas.

Considerando essa particularidade regional do desenvolvimento da pesquisa, sugere-se novas investigações com o intuito de fortalecer as reflexões acerca da Metodologia de Cuidado Humanitude para outros contextos.

\section{Referências}

Bardin, L. Análise de conteúdo. (2016). Pinheiro LA, Tradução. São Paulo: Edições 70.

Brasil. Ministério da Saúde. Conselho Nacional de Saúde. (2012). Resolução n. 466, de 12 de dezembro de 2012. Aprova as diretrizes e normas regulamentadoras de pesquisas envolvendo seres humanos. Brasília: Ministério da Saúde, 2012. Recuperado de: http://conselho.saude.gov.br/resolucoes/2012/Reso466.pdf.

Costa, M. O., Galvão, A. M., \& Baptista, M. G. J. (2014). Percepção de enfermeiros acerca da metodologia de trabalho: humanitude na gestão de cuidados em saúde. Revista Referência, 11(3), 26. http://hdl.handle.net/10198/11521.

Damaceno, D. G., Chirelli, M. Q., \& Lazarini, C. A. (2019). A prática do cuidado em instituição de longa permanência para idosos: desafio na formação dos profissionais. Rev. Bras. geriatr. gerontol. 22(1), e180197. https://doi.org/10.1590/1981-22562019022.180197.

Dantas, C., Melo, R., Boleto, A., Silva, F., \& Rodrigues, F. (2017). Projeto IdoVis Visitadores contribuem para um envelhecimento com mais afetos. Journal of Aging \& Innovation, 6(3), 47-58. http://www.journalofagingandinnovation.org/wp-content/uploads/5-visitadores.pdf.

Duarte, M. L. C., Silva, D. G., \& Bagatini, M. M. C. (2021). Enfermagem e saúde mental: uma reflexão em meio à pandemia do coronavírus. Rev. Gaúcha Enferm. 42(esp), e20200140. https://doi.org/10.1590/1983-1447.2021.20200140.

Faro, A., Bahiano, M. A., Nakano, T. C., Reis, C., Silva, B. F. P., \& Vitti, L. S. COVID-19 e saúde mental: a emergência do cuidado. (2020). Estud. psicol. (Campinas), 37, e200074. https://doi.org/10.1590/1982-0275202037e200074

Ferguson, N. M., Laydon, D., Nedjati Gilani, G., Imai, N., Ainslie, K., Baguelin, M., ... \& Ghani, A. C. (2020). Report 9: impact of non-pharmaceutical interventions (NPIs) to reduce COVID19 mortality and healthcare demand. Imperial College London. 1-20. Recuperado de: http://dx.doi. org/10.25561/77482

Figueiredo, A. M.G., Melo, R. C. C. P., \& Ribeiro, O. P. (2018). Humanitude care methodology: Difficulties and benefits from its implementation in clinical practice. Rev. Enferm. Ref. 4(17), 53-62. Recuperado de: https://doi.org/10.12707/RIV17063

Gineste, Y., \& Pellissier J. (2008). Humanitude: Cuidar e compreender a velhice. Lisboa, Portugal: Instituto Piaget

Henriques, L. V. L., Dourado, M. A. R. F., Melo, R. C. C. P., \& Tanaka, L. H. (2019). Implementation of the Humanitude Care Methodology: contribution to the quality of health care. Rev. Latino-Am. Enfermagem. 27, e3123. Recuperado de: DOI: 10.1590/1518-8345.2430-3123 
Henriques, L., Dourado, M., Melo, R., Araújo, J. \& Inácio, M. (2020) Methodology of Care Humanitude Implementation at an Integrated Continuing Care Unit: Benefits for the Individuals Receiving Care. Open Journal of Nursing, 10, 960-972. https://doi.org/10.4236/ojn.2020.1010067

Kang, L., Li, Y., Hu, S., Chen, M., Yang, C., Yang, B. X., Wang, Y., ... \& Liu, Z. (2020). The mental health of medical workers in Wuhan, China dealing with the 2019 novel coronavirus. Lancet Psychiatry, 7(3), e14. 10.1016/S2215-0366(20)30047-X

Lai, J., Ma, S., \& Wang, Y. Factors associated with mental health outcomes among health care workers exposed to Coronavirus Disease 2019. (2020). JAMA Netw Open. 3(3):e203976. doi: 10.1001/jamanetworkopen.2020.3976

Lima, G. K. M., Gomes, L. M. X., \& Barbosa, T. L. A. (2020). Qualidade de Vida no Trabalho e nível de estresse dos profissionais da atenção primária. Saúde em Debate, 44(126), 774-789. https://doi.org/10.1590/0103-1104202012614

Machado, M. H., Pereira, E. J., Neto, F. R. G. X., \& Wermelinger, M. C. M. W. (2020). Enfermagem em tempos da COVID-19 no Brasil: um olhar da gestão de trabalho. Enferm. Foco, 11(1), 32-39. http://biblioteca.cofen.gov.br/wp-content/uploads/2020/08/EnfermagemCovid19GestaoTrabalho.pdf.

Melo, R. C. C. P., Queirós, P. J. P., Tanaka, L. H., Salgueiro, N. R. M., Alves, R. E., Araújo, J. P. \& Rodrigues, M. A. (2017). State-of-the-art in the implementation of the Humanitude care methodology in Portugal. Rev. Enf. Ref. 4(13), 53-62. https://doi.org/10.12707/RIV17019.

Melo, R. C. C. P., Costa, P. J., Henriques, L. V. L., Tanaka, L. H., Queirós, P. J. P., \& Araújo, J. P. (2019). Humanitude in the humanization of elderly care: experience reports in a health service. Rev. Bras. Enferm. 72(3), 825-829. http://dx.doi.org/10.1590/0034-7167-2017-0363.

Moraes, E. N., Viana, L. G., Resende, L. M. H., Vasconcellos, L. S., Moura, A. S., Menezes, A., Mansano, N. H., \& Rabelo, R. (2020). Ciências \& Saúde Coletiva.. 25(9), 3445-3458. 10.1590/1413-81232020259.20382020

Oliveira, R. D. V. L. (2020) Um ensaio sobre a cegueia: covid-19 e a humanização das ciências da natureza. RIDH, 8(2):71-81. https://www3.faac.unesp.br/ridh/index.php/ridh/article/view/831.

Oliveira, E. N., Costa, M. S. A., Nascimento, P. I. F. V., Rodrigues, C. S., Andrade, C. S. G., Mendonça, J. M. F., ... \& Lima, G. F. (2020). Com a palavra os profissionais de saúde na linha de frente do combate à COVID-19. Reserach, Society and Development,9(8), e30985145. https://doi.org/10.33448/rsdv9i8.5145.

Pinto, M. G. A. (2019). Metodologia de cuidado humanitude: Impacto na redução de burnout dos cuidadores formais numa ERPI. (Dissertação de mestrado). Instituto Superior de Serviço Social do Porto, Porto, Portugal.

Roquete, F. F., Batista, C. C. R. F., \& Arantes, R. C. (2017). Demandas assistenciais e gerenciais das instituições de longa permanência para idosos: uma revisão integrativa (2004-2014). Rev. Bras. Geriatr. Gerontol, 20(2), 288-301. https://doi.org/10.1590/1981-22562017020.160053.

Salyers, M. P., Bonfils, K. A., Luther, L., Firmin, R. L., White, D. A., Adams, E. L., \& Rollins, A. L. (2017). The relationship between professional burnout and quality and safety in healthcare: A meta-analysis. J Gen Intern Med. 32(4), 475-82. https://dx.doi.org/10.1007/s11606-016-3886-9.

Shultz, C. C., Corrêa, K. I. D., Vaz, S. M. C., Colet, C. F., \& Stumm, E. M. F. (2020). Resiliência da equipe de enfermagem no âmbito hospitalar com enfase na pandemia COVID-19. Reserach, Society and Development, 9(11), e539119466. https://doi.org/10.33448/rsd-v9i11.9466.

Silva, S. M., Baptista, P., Silva, F. J., Almeida, M. C., \& Soares, R. A. (2020). Fatores relacionados à resiliência em trabalhadores de enfermagem no contexto hospitalar. Rev. esc. enfermagem USP, 54, e03550. http://dx.doi.org/10.1590/s1980-220x2018041003550.

Sunde, R. M., \& Sunde, L. M. C. (2020). Idosos asilados: atenção dos cuidadores e a contaminação por COVID-19. JCS HU-UFPI, 3(2):e-11097. https://doi.org/10.26694/jcs_hu-ufpi.v3i1.11097.

Tavares, C. V. L. B., Melo, R. C. C. P., \& Henriques, L. V. L. (2020). Desafios no cuidar: estratégias facilitadoras da internação com a pessoa idosa. Revista investigação em enfermagem, 33(2), p.45-51. https://www.sinaisvitais.pt/images/stories/Rie/RIE33_s2.pdf\#page=45.

Ventura-Silva, J. M. A., Ribeiro, O. M. P. L., Santos, M. R., Faria, A. C. A., Monteiro, M. A. J., \& Vandresen, L. (2020). Planejamento organizacional no contexto de pandemia por COVID-19: implicações para a gestão em enfermagem. Journal Health NPEPS, 5(1):e4626. http://dx.doi.org/10.30681/252610104626.

Xiang, Y. T., Yang, Y., Li, W., Zhang, L., Zhang, Q., Cheung, T., \& Ng, C. H. (2020). Timely mental health care for the 2019 novel coronavirus outbreak is urgently needed. Lancet Psychiatry. 7(3):228-9. 10.1016/S2215-0366(20)30046-8

World Health Organization (WHO). Coronavirus disease (COVID-19) pandemic [Internet]. (2020). Geneva: World Health Organization. https://www.who.int/emergencies/diseases/novel-coronavirus-2019

Yin, R. K. Pesquisa qualitativa do início ao fim. (2016). Porto Alegre: Penso.

Zhang, C., Yang, L., Liu, S., Ma, S., Wang, Y., Cai, Z., Du, H., ... Zhang, B. (2020). Survey of insomnia and related social psychological factors among medical staff involved in the 2019 novel coronavirus disease outbreak. Frontiers in Psychiatry, 11(306), 1-9. http://dx.doi.org/10.3389/fpsyt.2020.00306 